\title{
Detection and Evaluation of Degradation in Electric Wire Connection using Infrared Thermography
}

\author{
by Kotaro Yamakoshi", Naoyuki Kumagai", Hirotsugu Inoue ${ }^{* * *}$, Hirofumi Yamada*, Yutaka Kasai" \\ * Thermographers Co., Ltd. 1-4-14, Suge, Tama-ku, Kawasaki-shi, Kanagawa 214-0001, Japan, \\ yamakoshi@thgs.co.jp \\ ${ }^{* *}$ The Kansai Electric Power Company, Incorporated, Office of Renewable Energy Business Strategy, 3-6-16 \\ Nakanoshima, Kita-ku, Osaka 530-8270, Japan, kumagai.naoyuki@d4.kepco.co.jp \\ *** Tokyo Institute of Technology, Department of Mechanical Engineering, 2-12-1 O-okayama, Meguro, Tokyo \\ 152-8552, Japan, inoue.h.ab@m.titech.ac.jp
}

\begin{abstract}
Degradation detection of the electric wire connection (compression straight sleeves, compression type anchor clamps, etc.) of the electric power transmission line is usually conducted by directly measuring the resistance of the connection and judging by comparison with the wire resistance ratio. For the measurement, electric power supply to the transmission line is stopped; the worker climbs the power transmission tower and measures the resistance value. It is a time-consuming and dangerous work because of high place and accidental high voltage. If the degradation is accurately detected from the ground by using infrared thermography, it can contribute to work safety/efficiency, cost reduction, and stable electric power supply without planned power outage. In this study, we examined conditions, application range, accuracy and judgment method for detecting the degradation of wire connection quantitatively by using infrared thermography. We report the results of heat radiation test of the power line and investigation of the relationship between the measured data and the degradation.
\end{abstract}

\section{Introduction}

This research was conducted for two years from 2014 as a contract research from Kansai Electric Power Co., Ltd. Initially, the relationship between heat generation and deterioration of the transmission line was obtained experimentally in the laboratory. Then measurements were actually performed in the field based on the results obtained in the laboratory. It was verified that the relationship between heat generation and deterioration was appropriate to evaluate the deterioration of actual transmission line. In addition, a work manual was established based on this result.

\section{Principle of degradation detection by infrared thermography system}

Degradation of the connection part appears as an increase in electric resistance. Joule heat is generated in the live-line state to which electric power is supplied, so that it is possible to specify where the electric resistance is larger than normal by measuring the surface temperature. If the connection part deteriorates and the contact resistance increases, the calorific value increases. However, even if it is possible to detect the heat generation location by the thermography, there is no means for quantitatively evaluating the resistance value from the calorific value. A diagnostic method capable of quantitatively evaluating the degree of deterioration is required.

\section{Methodology}

\subsection{Basic experiment}

At first, basic data were acquired with considering the conditions, applicable range, accuracy, judgment method, etc. for detecting the degradation of the wire connection part by the infrared thermography apparatus. Then, the actual measurement was carried out several times for verification.

The relationship between the calorific value and the load current was determined by laboratory test. The power transmission member as shown in figure 1 was connected to the current load test device and the heat generation in the member was measured with changing the current load. The surface temperature of the connecting pipe was measured with infrared thermography and thermocouple. The correlation between current value, resistance value and temperature were examined. As a result, temperature rise according to Joule heat generation was recognized at each measurement location. In addition, it was found that the spatial gradient of the calorific value varies depending on the measurement location.

\subsection{Additional experiment}

Both the emissivity of the measuring object and the measurement distance limit (the optical performance 
evaluation of the equipment) was also evaluated because these are important factors affecting the temperature measurement. It was found that the emissivity is highest at the electric wire main line as shown in Table 1 and hence that the measurement should be conducted at the electric wire main line. For the spatial resolution, the slit response was measured for two products of Nippon Avionics and FLIR. The value at which the output becomes $90 \%$ is determined as substantial spatial resolution.
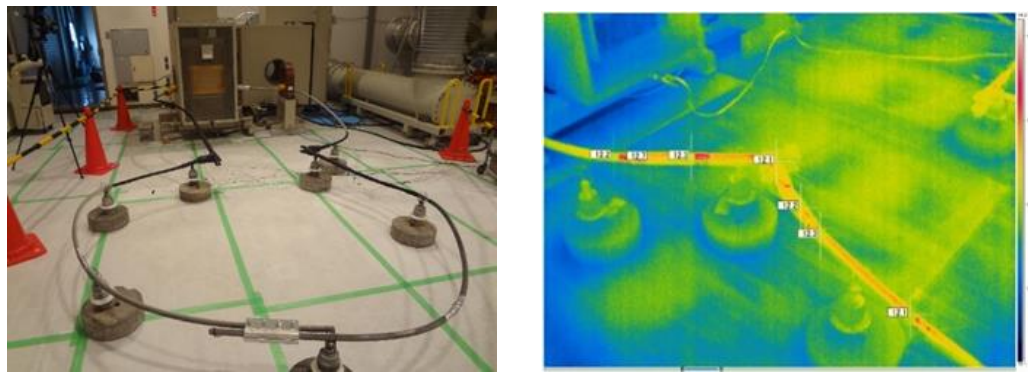

Figure 1: Test body connected in series with the current load test device. A thermocouple was attached to the connection part (Left). Thermography image of the connection part (right)

Table 1: Emissivity of each part

\begin{tabular}{|l|c|c|c|}
\hline \multicolumn{1}{|c|}{ Part } & $\begin{array}{c}\text { Black body painted temperature }\left({ }^{\circ} \mathrm{C}\right) \\
\text { (Emissivity setting 0.98) }\end{array}$ & $\begin{array}{c}\text { Surface of parts temperature }\left({ }^{\circ} \mathrm{C}\right) \\
\text { (Emissivity setting 1.0) }\end{array}$ & Emissivity \\
\hline a. Wire & 64.0 & 60.4 & $\mathbf{0 . 9 1}$ \\
\hline b. Main clamp & 47.1 & 33.1 & $\mathbf{0 . 5 6}$ \\
\hline c. Jumper clamp & 48.1 & 34.9 & $\mathbf{0 . 5 9}$ \\
\hline d. Jumper wire & 58.5 & 36.7 & $\mathbf{0 . 4 8}$ \\
\hline
\end{tabular}

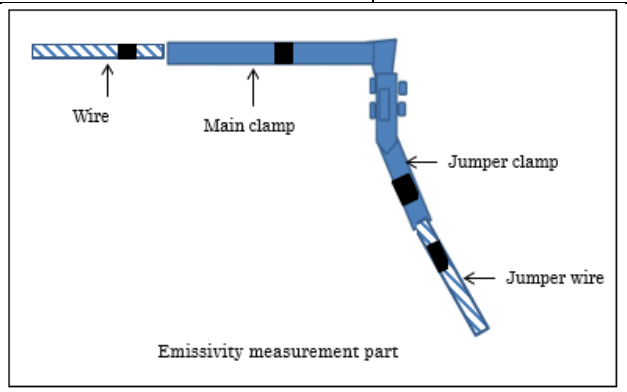

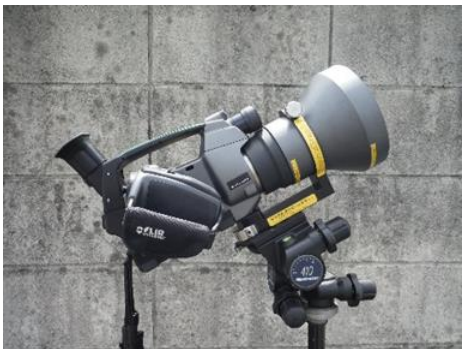

FLIR SC660+7\%ens

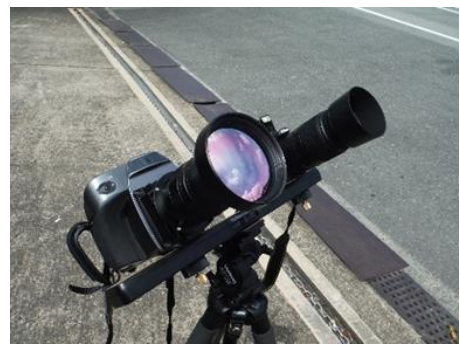

Nippon Avionics 500EX+3.3\%ens Figure 2: Measurement apparatus

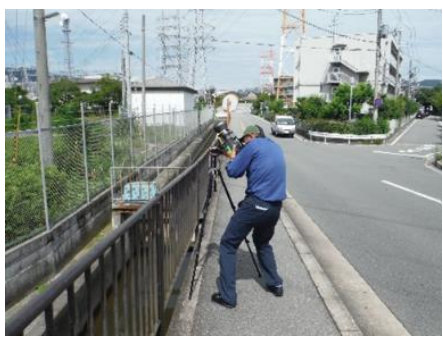

Measurement scenery

\subsection{Establishment of work manual}

In order to apply the technique developed by this research to on-site test, a work manual was established for transmission line connection part deterioration detection. The manual summarizes equipment used, measured distance, measurement time and so on.

\section{Future issues}

Field measurements were carried out in FY 2016. Degradation diagnosis manual was further established from the result of this field measurement. However, actual operation is not implemented yet because it is necessary to evaluate the influence of environment such as wind and humidity on measurement and its correction. In the future, measurements will be conducted according to the manual and then the diagnostic accuracy will be improved further. 